Article

\title{
Extra Virgin Olive Oil Phenol Extracts Exert Hypocholesterolemic Effects through the Modulation of the LDLR Pathway: In Vitro and Cellular Mechanism of Action Elucidation
}

\author{
Carmen Lammi ${ }^{1, *}$, Maria Bellumori ${ }^{2}{ }^{\oplus}$, Lorenzo Cecchi ${ }^{2}{ }^{\oplus}$, Martina Bartolomei ${ }^{1}$, \\ Carlotta Bollati ${ }^{1}$, Maria Lisa Clodoveo ${ }^{3}{ }^{\mathbb{D}}$, Filomena Corbo ${ }^{4}$ (D), Anna Arnoldi ${ }^{1}$ (D) \\ and Nadia Mulinacci ${ }^{2}$ \\ 1 Department of Pharmaceutical Sciences, University of Milan, 20133 Milan, Italy; \\ martina.bartolomei@studenti.unimi.it (M.B.); carlotta.bollati@unimi.it (C.B.); anna.arnoldi@unimi.it (A.A.) \\ 2 Department of Neuroscience, Psychology, Drug and Child Health, Pharmaceutical and Nutraceutical Section, \\ University of Florence, 50019 Florence, Italy; maria.bellumori@unifi.it (M.B.); lo.cecchi@unifi.it (L.C.); \\ nadia.mulinacci@unifi.it (N.M.) \\ 3 Interdisciplinar Department of Medicine, University Aldo Moro Bari, 70125 Bari, Italy; \\ marialisa.clodoveo@uniba.it \\ 4 Department of Pharmacy-Pharmaceutical Sciences, University Aldo Moro Bari, 70125 Bari, Italy; \\ filomena.corbo@uniba.it \\ * Correspondence: carmen.lammi@unimi.it; Tel.: +39-025-031-9372
}

Received: 20 May 2020; Accepted: 4 June 2020; Published: 9 June 2020

\begin{abstract}
This study was aimed at investigating the hypocholesterolemic effects of extra virgin olive oil (EVOO) phenols and the mechanisms behind the effect. Two phenolic extracts were prepared from EVOO of different cultivars and analyzed using the International Olive Council (IOC) official method for total phenols, a recently validated hydrolytic procedure for total hydroxytyrosol and tyrosol, and ${ }^{1} \mathrm{H}-\mathrm{NMR}$ analysis in order to assess their secoiridoid profiles. Both of the extracts inhibited in vitro the 3-hydroxy-3-methylglutaryl co-enzyme A reductase (HMGCoAR) activity in a dose-dependent manner. After the treatment of human hepatic HepG2 cells $(25 \mu \mathrm{g} / \mathrm{mL})$, they increased the low-density lipoprotein (LDL) receptor protein levels through the activation of the sterol regulatory element binding proteins (SREBP)-2 transcription factor, leading to a better ability of HepG2 cells to uptake extracellular LDL molecules with a final hypocholesterolemic effect. Moreover, both of the extracts regulated the intracellular HMGCoAR activity through the increase of its phosphorylation by the activation of AMP-activated protein kinase (AMPK)-pathways. Unlike pravastatin, they did not produce any unfavorable effect on proprotein convertase subtilisin/kexin 9 (PCSK9) protein level. Finally, the fact that extracts with different secoiridoid profiles induce practically the same biological effects suggests that the hydroxytyrosol and tyrosol derivatives may have similar roles in hypocholesterolemic activity.
\end{abstract}

Keywords: EVOO phenols; HepG2 cells; hypocholesterolemic; PCSK9; LDL receptor

\section{Introduction}

Cardiovascular disease (CVD) is a leading cause of death worldwide and hypercholesterolemia is one of the main risk factors responsible for the development of this multifactorial disease. The low-density lipoprotein (LDL) fraction transports the majority of plasma cholesterol, and the hepatic LDL receptor (LDLR) is responsible for the cellular LDL-uptake and catabolism [1,2]. In general, the LDLR expression is finely tuned by changes in intracellular cholesterol and a transcription factor, known 
as the sterol-responsive element binding protein-2 (SREBP-2), plays a critical role in LDLR mRNA expression [3,4]. Among SREBP-2 gene targets, the 3-hydroxy-3-methylglutaryl coenzyme A reductase (HMGCoAR) is particularly important [5]. This enzyme plays a key role in the intracellular cholesterol biosynthesis, since it is the rate controlling enzyme in the mevalonate pathway, which is also regulated by the AMP-activated protein kinase (AMPK) pathway [3]. More in details, the LDLR expression and receptor protein localization at cellular membranes are strictly correlated to the intracellular cholesterol biosynthesis pathway. In fact, the transcription of the LDLR and the genes that are required for cholesterol and fatty acid synthesis are controlled by membrane-bound transcription factors called SREBPs [3], and the intracellular cholesterol acts with a negative feedback inhibition mechanism. The SREBP-2 isoform is responsible for the LDLR and HMGCoAR transcription, and the SREBP-2 maturation is regulated by the intracellular cholesterol homeostasis. In fact, after synthesis, SREBP-2 forms a complex with the SREBP cleavage-activating protein (SCAP) and it is localized in the endoplasmic reticulum (ER) as an inactive precursor (pro-SREBP-2). Sterol deficiency results in the release of SREBP-2/SCAP complex from ER and transport to the Golgi, where pro-SREBP2 is processed further, allowing for it to enter the nucleus and up-regulate transcription of LDLR and HMGCoAR [1-3]. The increase of LDLR determines an increased clearance of plasmatic LDL-cholesterol with a reduction of cholesterolemia, one of the main risk factors for CVD progression.

In addition, the activity of the LDLR is regulated by proprotein convertase subtilisin/kexin type 9 (PCSK9) at the post-transcriptional level [6,7]. Briefly, PCSK9 and LDLR both contain functional SREs in their promoters that respond to change in intracellular cholesterol levels through the activation of the SREBP pathway. However, since the HNF1-alpha binding site is unique to the PCSK9 promoter and it is not present in the LDLR promoter, the modulation of PCSK9 transcription through HNF1-alpha sequence does not affect LDLR gene expression. Thus, the HNF1-alpha binding site represents a divergent point to disconnect the co-regulation of PCSK9 from LDLR and other SREBP target genes.

Through its functional ability to bind the LDLR, PCSK9 prevents receptor recycling on the hepatocytes surface and promotes its lysosomal degradation, leading to increased LDL-C levels. For this reason, PCSK9 is a promising alternative target for developing new hypocholesterolemic drugs [8]. It is important to underline that statins increase the PCSK9 expression, which dampens effective LDL clearing by promoting LDLR degradation [9], thereby counteracting the therapeutic effects of these drugs.

Extra virgin olive oil (EVOO) is an unrefined oil that is known for its healthy properties, mainly in the area of CVD prevention. Numerous studies have shown that EVOO reduces blood pressure [10], improves the lipid profile by increasing HDL-cholesterol and reducing LDL-cholesterol and triglyceride levels [11,12], reduces oxidative stress, and inhibits human lipoprotein oxidation, which makes LDL less atherogenic [11,13]. These health benefits are often attributed to the high oleic acid content; however, unlike all other vegetable oils, EVOO contains high amounts of peculiar bioactive molecules, including phenolic compounds comprising the group of secoiridoids, as derivatives of oleuropein and ligstroside, as well as smaller amounts of free hydroxytyrosol and tyrosol [14].

The first objective of present study was an investigation of the hypocholesterolemic effects of EVOO phenols and the mechanisms behind this effect. As a second objective, the study aimed to find a relationship between the phenolic profiles and observed activities. For this reason, the investigation was conducted on the phenolic extracts that were obtained from two different cultivars. The former was extracted from the EVOO of cultivar Frantoio cultivated in Tuscany (Italy) and was named BUO, whereas the latter was extracted from the EVOO of cultivar Coratina cultivated in Apulia (Italy) and was named OMN. The detailed phenolic profiles of the two extracts were obtained using the International Olive Council (IOC) official method for total phenols, a recently validated hydrolytic procedure for total hydroxytyrosol and tyrosol, and ${ }^{1} \mathrm{H}-\mathrm{NMR}$ analysis for the main secoiridoid aldehydes.

The beneficial effects of EVOO phenols may be mediated via a plethora of biochemical pathways and signaling mechanisms that may act either independently or synergistically. For this reason, after the first positive observations, a deeper mechanistic investigation was undertaken in order to 
find out how EVOO phenols may modulate the activity of the key targets involved in cholesterol metabolism; i.e., LDLR, SREBP-2, HMGCoAR, and PCSK9. To achieve this goal, HepG2 cells were treated with BUO and OMN extracts and their ability to modulate the LDLR pathway was investigated while using a combination of molecular and functional techniques.

\section{Materials and Methods}

\subsection{Chemicals}

All of the reagents and solvents employed were from commercial sources. See "Supplementary Materials" for further details on materials and methods.

\subsection{Selection of the Extra Virgin Olive Oils}

The study was conducted on two EVOO extra virgin olive oil samples, which were collected in the 2017 olive oil campaign. The former was produced by Società Agricola Buonamici SrL (Fiesole, Florence, Italy) and was a monocultivar sample from the typical Tuscan cultivar Frantoio (extract name BUO). The latter was from Azienda Agricola Donato Conserva (Modugno, Bari, Italy) and it was from the typical Apulian cultivar Coratina (extract name OMN). The oils were initially analyzed according to the official analytical methods described in Regulation EEC/2568/91 and further amendments and additions [15], for confirming that they belonged to the extra virgin category.

\subsection{HPLC-DAD Analysis of Phenols from EVOO}

Phenolic compounds were analyzed both before and after acid hydrolysis [16]. The phenols extraction was carried out according to the IOC method [17] in the presence of syringic acid as internal standard. The chromatographic analyses were carried out with a HP 1100 system that was provided with a quaternary pump and a DAD detector (Agilent Technologies, Santa Clara, CA, USA). Phenols were separated using a SphereClone ODS (2), $5 \mu \mathrm{m}, 250 \times 4.6 \mathrm{~mm}$ id column; the elution was obtained by $\mathrm{H}_{2} \mathrm{O}$ (at $\mathrm{pH} 2.0$ by phosphoric acid), acetonitrile and methanol as eluents, while applying the gradient reported in the IOC method (IOC/T.20/Doc No. 29); flow rate, $1 \mathrm{~mL} / \mathrm{min}$, injection volume $20 \mu \mathrm{L}$. The chromatograms were registered at $280 \mathrm{~nm}$ and syringic acid was used as an internal standard for the quantitative analysis, thus expressing the results as $\mathrm{mg}$ tyrosol $/ \mathrm{kg}$ oil.

The hydroalcoholic extracts obtained as described above were treated by the acid hydrolysis method that was previously proposed in order to evaluate the total contents of free and bound tyrosol and hydroxytyrosol [16]. Briefly, $300 \mu \mathrm{L}$ of the extract were heated at $80{ }^{\circ} \mathrm{C}$ for $2 \mathrm{~h}$ in the presence of $300 \mu \mathrm{L}$ of $\mathrm{H}_{2} \mathrm{SO}_{4} 1.0 \mathrm{M}$, and the solution was then diluted with $400 \mu \mathrm{L}$ of water. The following chromatographic analysis was carried out in a HP1200 liquid chromatograph that was equipped with a DAD detector (Agilent Technologies, Santa Clara, CA, United States) and a reverse phase (RP) C18 column, $150 \times 3 \mathrm{~mm}(5 \mu \mathrm{m})$ Gemini (Phenomenex, Torrance, CA, USA); flow rate, $0.4 \mathrm{~mL} / \mathrm{min}$. Eluents: $\mathrm{H}_{2} \mathrm{O}$ acidified to $\mathrm{pH} 3.2$ with formic acid (A) and acetonitrile (B). The linear solvent gradient was applied, as follows: solvent A varied $95 \%$ to $70 \%$ in $5 \mathrm{~min}$., then to $50 \%$ in $5 \mathrm{~min}$., then varied to $2 \%$ in 5 min., and stayed in this condition for 5 more min.; finally, solvent A came back to $95 \%$ in $2 \mathrm{~min}$. The total time of analysis was $22 \mathrm{~min}$., equilibration time, $10 \mathrm{~min}$. The total content of tyrosol was evaluated using a calibration line built using an authentic standard (purity grade 98\%), and considering the chromatographic areas at $280 \mathrm{~nm}$. Regarding hydroxytyrosol, its amount was evaluated again while using the calibration line of tyrosol at $280 \mathrm{~nm}$, but applying the following formula for keeping into account that it is overestimated by about $35 \%$ : $\mathrm{mg} \mathrm{OH}$-tyrosol $=\mathrm{mg}$ tyrosol $\times 0.65$ [18]. All data were expressed as $\mathrm{mg} / \mathrm{kg}$ oil.

\subsection{Preparation of the Phenolic Extracts for the Biological Testing}

Phenolic compounds were extracted from the two EVOO samples according to the following procedure: $50 \mathrm{~g}$ of each EVOO sample were exactly weighted and then put in a $500 \mathrm{~mL}$ flask together 
with $150 \mathrm{~mL}$ of $\mathrm{MeOH}: \mathrm{H}_{2} \mathrm{O}$ 80:20 solution. The mixture was vigorously hand-shaken for $1 \mathrm{~min}$. The extraction was then performed with the aid of an ultrasound bath for $15 \mathrm{~min}$. The obtained mixture was centrifuged at $5000 \mathrm{rpm}$ for $25 \mathrm{~min}$; the supernatant was recovered and filtered using PVDF type $0.45 \mu \mathrm{m} 13 \mathrm{~mm}$ diameter filters with a $60 \mathrm{~mL}$ syringe. The obtained solution was defatted twice with $75 \mathrm{~mL}$ of hexane and then evaporated under vacuum at room temperature. The dried extract was then dissolved in ethanol up to a total volume of $10 \mathrm{~mL}$. The obtained solution was then split in 10 vials, the solvent evaporated, thus obtaining 10 aliquots of dried extract (each corresponding to $5 \mathrm{~mL}$ of EVOO), to be used for the chemical and biological analysis. By this method, each BUO vial contained approximately $10.1 \pm 0.16 \mathrm{mg}$ dry weight, whereas each OMN vial $5.6 \pm 0.12 \mathrm{mg}$ of dry weight.

\subsection{Analysis of the Phenolic Extract}

The samples that were prepared for the biological testing were submitted to the HPLC-DAD analysis, as indicated above, as well as to ${ }^{1} \mathrm{H}-\mathrm{NMR}$ analysis using a literature method [19], by using a $400 \mathrm{MHz}$ instrument Advance 400 (Bruker, Bremen, Germany). For the NMR analysis, each defatted phenolic extract, which was obtained from $5 \mathrm{~mL}$ of EVOO, was dissolved in $1 \mathrm{~mL}$ of $\mathrm{CDCl}_{3}$.

\subsection{Cell Culture Conditions and Treatment}

HepG2 cell line was bought from ATCC (HB-8065, ATCC from LGC Standards, Milan, Italy) and cultured following standard protocol. More details are reported in the Supplementary Materials.

BUO and OMN extracts were tested separately. Briefly, each EVOO extract was diluted using DMSO in order to prepare a stock solution $(50 \mathrm{mg} / \mathrm{mL})$, which was diluted to reach the final concentration of $25.0 \mathrm{ug} / \mathrm{mL}$ in complete growth DMEM. The growth medium of adherent HepG2 cells was discarded and each diluted EVOO extract in complete DMEM was replaced and then incubated for the desirable incubation time based on the experiments.

\subsection{MTT Assay}

A total of $3 \times 10^{4}$ HepG2 cells/well were seeded in 96-well plates and treated with 25, 50, 100, and $200 \mu \mathrm{g} / \mathrm{mL}$ of BUO and OMN EVOO extracts, or vehicle $\left(\mathrm{H}_{2} \mathrm{O}\right)$ in complete growth media for $48 \mathrm{~h}$ at $37{ }^{\circ} \mathrm{C}$ under $5 \% \mathrm{CO}_{2}$ atmosphere. Subsequently, the solvent was aspirated and 3-(4,5-dimethylthiazol-2-yl)-2,5-diphenyltetrazolium bromide (MTT) assay was performed following conditions that are detailed reported in Supplementary Materials.

\subsection{HMGCoAR Activity Assay}

The assay buffer, NADPH, substrate solution, and HMGCoAR were provided in the HMGCoAR Assay Kit (Sigma). The experiments were carried out following the manufacturer's instructions and conditions that were previously optimized at $37^{\circ} \mathrm{C}$ [20]. More detailed information is reported in Supplementary Materials.

\subsection{Western Blot Analysis}

The experiments were performed following conditions previously described [21]. In particular, a total of $1.5 \times 10^{5} \mathrm{HepG} 2$ cells/well (24-well plate) were treated with $25.0 \mu \mathrm{g} / \mathrm{mL}$ of BUO or OMN EVOO extracts or pravastatin $1.0 \mu \mathrm{M}$ for $24 \mathrm{~h}$. After each treatment, the cells were processed for western blotting analysis following conditions that were already optimized and described [20]. More details are available on Supplementary Materials.

\subsection{In-Cell Western (ICW) Assay}

Experiments were performed following the previously described conditions by us elsewhere [22]. Briefly, a total of $3 \times 10^{4}$ HepG2 cells/well were seeded in 96-well plate and, the following day, they were treated with $25 \mu \mathrm{g} / \mathrm{mL}$ of BUO and OMN extracts or Pravastatin $1.0 \mu \mathrm{M}$ in complete growth 
medium for $24 \mathrm{~h}$. Subsequently, ICW assay was carried out following the protocol that is available on Supplementary Materials.

\subsection{Ffluorescent LDL Uptake}

Experiments were carried out following condition already described [23]. Briefly, a total of $3 \times$ $10^{4}$ HepG2 cells/well were seeded in 96-well plates and then kept in complete growth medium for $2 \mathrm{~d}$ before treatment. On the third day, LDL-Uptake was carried out following protocol that is detailed described in the Supplementary Materials.

\subsection{Statistical Analysis}

Statistical analyses were carried out by t-student and One-way ANOVA and Graphpad Prism 7, followed by Brown-Forsythe's test. Values were expressed as means \pm S.D.; $p$-values $<0.05,0.01$, 0.001 , and 0.0001 were considered to be significant.

\section{Results}

\subsection{Characterization of The Phenolic Extracts}

The two monocultivar oils were selected as representative of the high quality EVOO category. Their phenolic contents were determined according to the IOC method and after acidic hydrolysis. This latter method recently validated and applied to approximately 100 EVOO samples [16] was used in this study to obtain more details on the oleuropein and ligstroside derivatives of the two extracts. Indeed, the method allowed for measuring the total content of tyrosol and hydroxytyrosol released during the acid hydrolysis from ligstroside and oleuropein derivatives, respectively (EVOO columns in Table 1). The main differences between the two extracts were that BUO was richer in oleuropein derivatives $(444.9 \mu \mathrm{g} / \mathrm{g}$, measured as total hydroxytyrosol after hydrolysis) and OMN was richer in ligstroside derivatives $(308.6 \mu \mathrm{g} / \mathrm{g}$, measured as total tyrosol after hydrolysis).

Table 1. Phenolic content in the BUO and OMN samples (extra virgin olive oils (EVOOs) and dry extracts) before and after acid hydrolysis expressed in $\mu \mathrm{g} / \mathrm{g}$ in the case of the EVOO and in $\mu \mathrm{g} / \mathrm{mg}$ in the case of the dry extracts.

\begin{tabular}{ccccc}
\hline \multicolumn{5}{c}{ Phenolic Content } \\
\hline & \multicolumn{3}{c}{ BUO (before Hydrolysis) } & OMN (before Hydrolysis) \\
\hline Free hydroxytyrosol & $9.3 \pm 0.9$ & $4.0 \pm 0.1$ & $17.4 \pm 5.1$ & $16.0 \pm 4.6$ \\
Free tyrosol & $5.1 \pm 0.1$ & $2.0 \pm 0.1$ & $15.6 \pm 5.4$ & $14.0 \pm 0.8$ \\
Total Phenols & $617.9 \pm 34.1$ & $289.3 \pm 15.6$ & $415.7 \pm 7.3$ & $371.1 \pm 7.5$ \\
\hline & \multicolumn{7}{c}{ BUO (after Hydrolysis) } & OMN (after Hydrolysis $)$ \\
\hline Total hydroxytyrosol & $444.9 \pm 33.4$ & $208.0 \pm 15.6$ & $169.6 \pm 2.6$ & $151.0 \pm 3.0$ \\
Total tyrosol & $332.9 \pm 7.9$ & $156.0 \pm 3.9$ & $308.6 \pm 2.7$ & $275.1 \pm 2.7$ \\
Tyr + OH-tyr & $777.8 \pm 41.3$ & $364.1 \pm 19.5$ & $478.1 \pm 5.3$ & $426.1 \pm 5.7$ \\
\hline
\end{tabular}

Data are expressed as mean \pm SD of three replicates.

The samples for the biological tests were prepared, starting from $50 \mathrm{~mL}$ of each oil, and were accurately defatted with n-hexane. Taking the low water stability of most of the extracted phenols into account, each extract was divided in ten aliquots, dried, and stored at $-22{ }^{\circ} \mathrm{C}$ until use. Specifically, $10.1 \mathrm{mg}$ of phenol extract were obtained from $5 \mathrm{~mL}$ of BUO oil, whereas $5.6 \mathrm{mg}$ from $5 \mathrm{~mL}$ of OMN oil. These dried extracts were again submitted to HPLC (dry extracts in Table 1 ) as well as to ${ }^{1} \mathrm{H}-\mathrm{NMR}$ analysis (Table 2). The total phenolic contents of the two extracts were significantly different, with the OMN extract being the richest one either before $(371.1 \pm 7.5 \mu \mathrm{g} / \mathrm{mg})$ or after hydrolysis $(426 \pm 12.8 \mu \mathrm{g} / \mathrm{mg})$. 
These differences, with respect to the original EVOOs (that showed an inverse situation), can be explained by the diverse dry weights of the extracts that were obtained starting from the same volume of oil.

Table 2. Ratio of integral of signals related to specific monoaldehydic forms of secoiridoids in ${ }^{1} \mathrm{H}-\mathrm{NMR}$ spectra of the dry extracts from BUO and OMN samples.

\begin{tabular}{ccc}
\hline Integral Ratio $\left({ }^{\mathbf{1}} \mathbf{H}-\mathrm{NMR}\right)$ & & \\
\hline & BUO & OMN \\
Oleacein/Oleocanthal & 1.09 & 0.49 \\
Oleur A./Ligstr. A & 1.73 & 0.60 \\
Oleacein + Oleur. A/Oleochantal + Ligstr. A & 1.4 & 0.58 \\
\hline Oleur. A, aglycone of oleuropein; Ligstr. A. aglycone of ligstroside.
\end{tabular}

Oleur. A, aglycone of oleuropein; Ligstr. A. aglycone of ligstroside.

As concerning the specific secoiridoids content (measured after hydrolysis), hydroxytyrosol was predominant in the BUO extract $(208.0 \pm 15.6 \mu \mathrm{g} / \mathrm{g}$ dried extract), while tyrosol was more abundant in the OMN one $(275.1 \pm 2.7 \mu \mathrm{g} / \mathrm{g}$ dried extract $) . \quad{ }^{1} \mathrm{H}-\mathrm{NMR}$ analysis was performed on both extracts in order to measure specific ratios between the four main secoiridoids of EVOO, namely oleacein, oleuropein aglycone, oleocanthal, and ligstroside aglycone. The specific ratios were calculated (Table 2) by measuring the integral of the duplets corresponding to the aldehyde protons of the selected molecules in the range between 9.20 and 9.60 ppm (i.e., oleacein, 9.23 ppm; oleocanthal, 9.60 ppm; oleuropein aglycone, 9.55 ppm; ligstroside aglycone, 9.53 ppm) [19]. These ratios highlight the significant differences in terms of secoiridoid molecules between the two extracts, which confirm that the oleuropein derivatives were widely prevalent in the BUO sample, in agreement with the results of the HPLC analysis of the hydrolyzed samples (Table 1).

\subsection{The Phenolic Extracts Drop In Vitro the HMGCoAR Activity}

Biochemical investigation was carried out for assessing the phenolic extract capacities to influence the HMGCoAR activity. The results clearly suggest that both extracts inhibit the enzyme activity with dose response trends (Figure 1). In particular, at the concentrations of 10.0, 50.0, 100.0, and 250.0 $\mu \mathrm{g} / \mathrm{mL}$, the BUO extract drops it by $5.4 \pm 1.1 \%, 39.1 \pm 10.6 \%, 48.0 \pm 6.0 \%$, and $74.8 \pm 7.2 \%$, respectively (Figure 1A), whereas the OMN extract reduces the enzyme activity by $18.2 \pm 0.6 \%, 22.7 \pm 6.0 \%$, $43.3 \pm 1.4 \%$, and $64.4 \pm 5.4 \%$, respectively (Figure $1 \mathrm{~B}$ ).

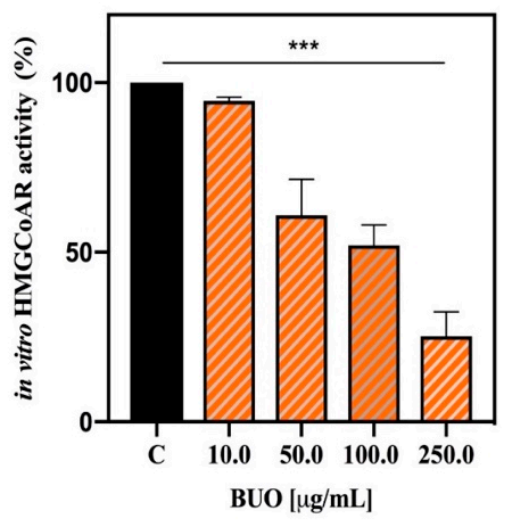

(A)

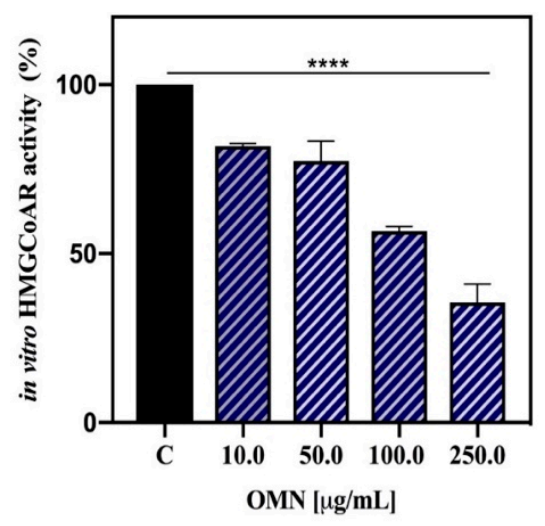

(B)

Figure 1. Effect of the phenolic extracts on the modulation of the in vitro HMGCoAR activity. Both BUO (A) and OMN (B) extracts drop HMGCoAR with dose-response trends. Data represent the mean \pm s.d. of six independent experiments performed in triplicate. $\left.{ }^{* * *}\right) p<0.001 ;\left({ }^{* * * *}\right) p<0.0001$. C: control sample. 


\subsection{Effects of the Phenols Extracts on the HepG2 Cell Vitality}

Cellular viability experiments were carried out for sorting out those concentrations of the OMN and BUO extract that may potentially produce cytotoxic effects on HepG2 cells. After a $48 \mathrm{~h}$ treatment, no cytotoxic effect was observed up to $100 \mu \mathrm{g} / \mathrm{mL}$ versus control cells (C), which suggests that neither BUO nor OMN extracts mediate a cytotoxic effect in this dose range, whereas, at $200 \mu \mathrm{g} / \mathrm{mL}, 31.5 \pm 2.4 \%$ and $16.8 \pm 2.7 \%$ cell mortalities were observed, respectively (Figure 2A,B). Thus, the following cellular investigations were performed at concentrations that were equal to $25.0 \mu \mathrm{g} / \mathrm{mL}$.

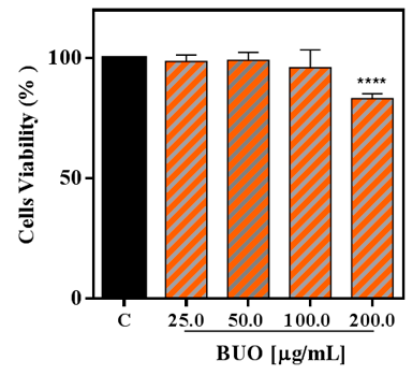

(A)

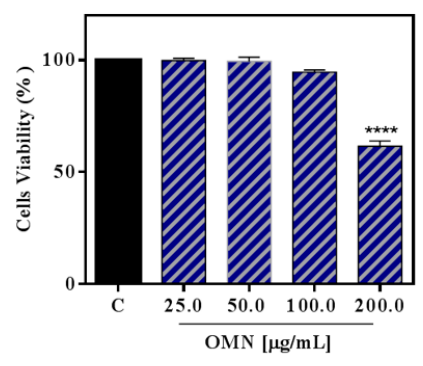

(B)

Figure 2. Cell vitality after treatment with phenol extracts. Both BUO (A) and OMN (B) samples did not affect the HepG2 vitality after $48 \mathrm{~h}$ of incubation up to $100 \mu \mathrm{g} / \mathrm{mL}$. The first cytotoxic effect was observed after the treatment of the hepatic cells with $200 \mu \mathrm{g} / \mathrm{mL}\left(^{* * * *}\right) p<0.0001$. Data represent the mean \pm s.d. of three independent experiments performed in triplicate C. untreated HepG2 cells.

\subsection{The Phenol Extracts Modulate the LDLR Pathways}

Human hepatic HepG2 cells were treated with both extracts at $25.0 \mu \mathrm{g} / \mathrm{mL}$ concentrations to evaluate the ability of the BUO and OMN phenol extracts to modulate the cholesterol metabolism. In parallel, HepG2 cells were treated with pravastatin $(1 \mu \mathrm{M})$ as reference control. Immunoblotting experiments were carried out on cell lysates. Figure $3 \mathrm{~A}-\mathrm{C}$ clearly indicate that the LDLR pathway is activated after both treatments. More in details, the BUO and OMN extracts up-regulate the protein level of the SREBP-2 transcription factor by $172 \% \pm 38.6 \%$ and $212 \pm 56.4 \%(p<0.05)$, respectively (Figure $3 \mathrm{~A}$ ), and the increase of SREBP-2 protein level lead to an improvement of total LDLR protein levels up to $153 \pm 24.4 \%$ and $177 \pm 28.3 \%(p<0.001)$, respectively (Figure 3B). These results are in agreement with the augmentation of LDLR population localized on the hepatocyte surface, which was assessed by an ICW assay. An improvement of the membrane LDLR levels up to $183 \pm 36.2 \%$ and $177 \pm 24.5 \%$ $(p<0.0001)$ was observed (Figure 3C). In the same set of experiments, at $1 \mu \mathrm{M}$, the positive control pravastatin increased the SREBP-2 by $156 \pm 4.6 \%(p<0.001)$, the LDLR protein levels by $152 \pm 19.7 \%$ $(p<0.05)$ (Figure 3A,B), and the membrane LDLR protein level by $50 \pm 1.4 \%$ (Figure 3C).

\subsection{The Phenol Extracts Modulate the HMGCoAR Activation by AMPK-Pathway Regulation}

For evaluating both phenol extract effects on the HMGCoAR protein levels, the HepG2 cells were treated with the extracts $(25.0 \mu \mathrm{g} / \mathrm{mL})$ for $24 \mathrm{~h}$ and suitable western blotting experiments were assessed. Upon SREBP-2 transcription factor augmentation, an improvement of the LDLR protein levels was observed as well as an increase of the HMGCoAR protein levels. As shown by Figure 4A, the HMGCoAR protein levels were enhanced by $163 \pm 25.6 \%$ and $124 \pm 15.3 \%$, respectively, while pravastatin $(1 \mu \mathrm{M})$ increased the enzyme protein levels up to $236.4 \pm 23.2 \%$ (Figure $4 \mathrm{~A}$ ). Moreover, the treatment with the BUO and OMN extracts significantly increased the phosphorylation levels of HMGCoAR (serine 872, AMPK phosphorylation site) up to $152 \pm 29.6 \%$ and $148 \pm 10.7 \%(p<0.05)$, respectively (Figure $4 \mathrm{~B}$ ). This result is in line with the improvement of AMPK phosphorylation (threonine 172) up to $146 \pm 16.4 \%(p<0.05)$ and $128 \pm 23.5 \%(p<0.05)$, respectively (Figure $4 \mathrm{C})$. 

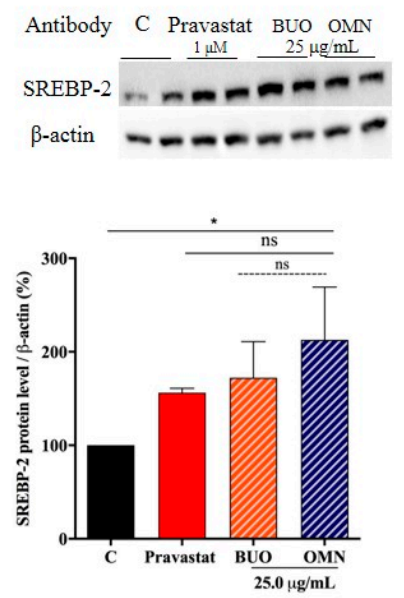
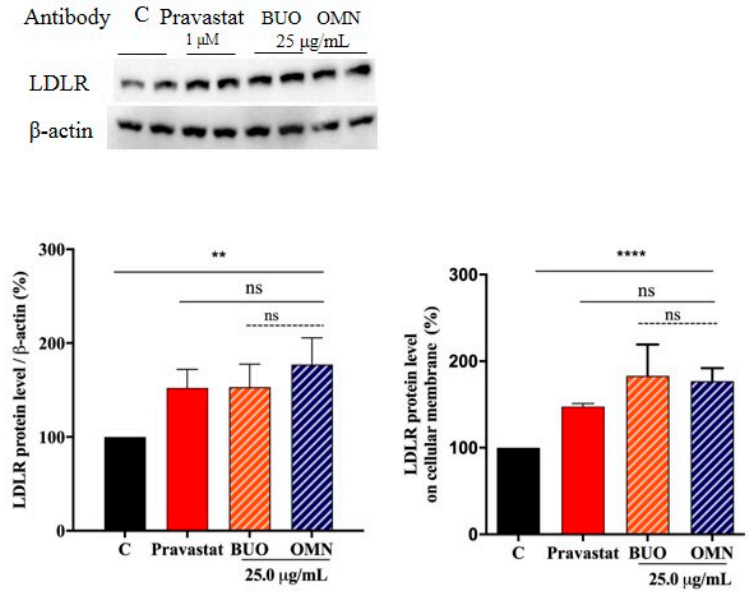

Figure 3. Modulation of the low-density lipoprotein receptor (LDLR) pathway by the phenol extracts. BUO and OMN extracts modulate the intracellular cholesterol pathway through the augmentation of SREBP-2 transcription factor protein level (A), which leads to the increase of the LDLR protein levels (B). Using In-Cell Western (ICW) assay, the BUON and OMN induction of LDLR localized on the cellular membrane of HepG2 cells is detected (C). The experiments were performed using in parallel pravastatin (Pravastat $1.0 \mu \mathrm{M}$ ) as positive control $(\mathbf{A}-\mathbf{C})$. Data represent the mean \pm s.d. of eight independent experiments performed in duplicate. $\left(^{*}\right) p<0.05,\left({ }^{* *}\right) p<0.01,\left({ }^{* * * *}\right) p<0.0001$. C. untreated HepG2 cells; Pravastat: pravastatin.

A
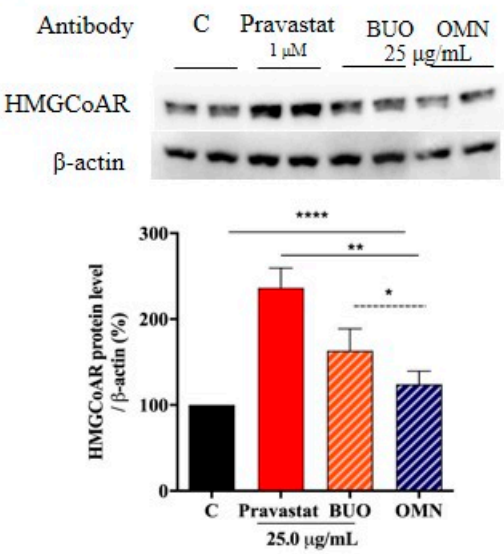

Antibody

D

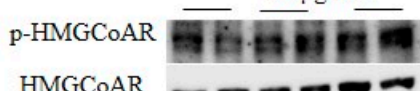

HMGCoAR

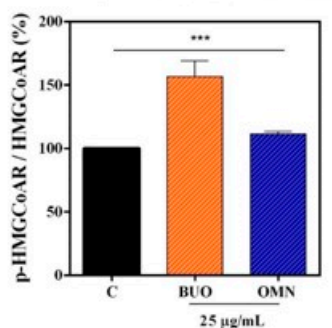

B
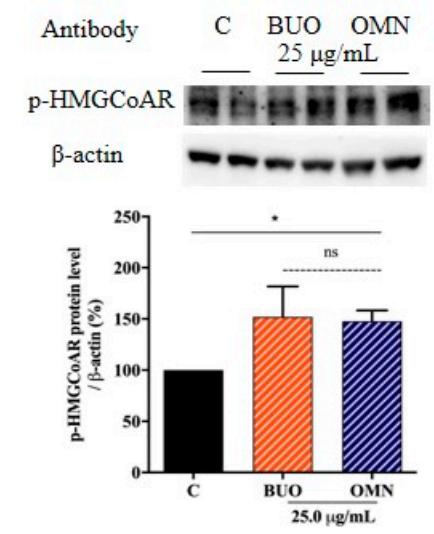

C
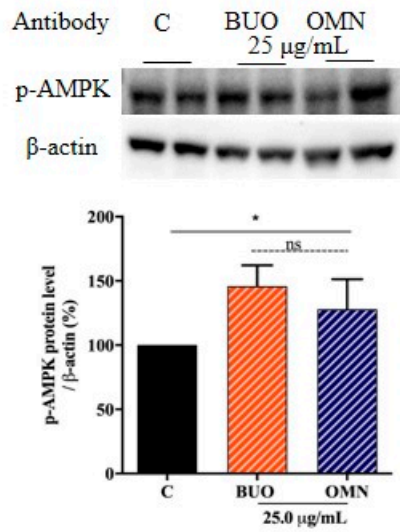

Figure 4. Regulation of the AMPK pathway. Both BUO and OMN samples increase the HMGCoAR protein levels, similarly to pravastatin used as a positive control (A). In addition, both extracts induce 
the activation of AMPK, through the augmentation of its phosphorylation on Thr172 residue, which in turn leads to the increase of the inactive phosphorylated HMGCoAR (p-HMGCoAR) protein levels (B-C). The ratio between p-HMGCoaR and total HMGCoAR was calculated after treatment with both BUO and OMN samples versus C sample (D). Data represent the mean \pm s.d. of eight independent experiments performed in duplicate. ${ }^{*}(p)<0.05,\left({ }^{* *}\right) p<0.01,\left({ }^{* * *}\right) p<0.001,\left({ }^{* * * *}\right) p<0.0001$. C. untreated HepG2 cells.

The pHMGCoAR/total HMGCoAR ratios of treated and untreated cells were also calculated. The ratio of treated cells was higher than that of untreated ones. In fact, the BUO and OMN extracts increased it up to $157 \pm 12.2 \%$ and $111 \pm 2.0 \%$, respectively $(p<0.001)$ (Figure $4 \mathrm{D})$.

\subsection{The Phenol Extracts Ameliorate HepG2 Ability to Absorb LDL}

Functional investigations were performed that aimed to evaluate whether the phenol extracts were able to modulate the HepG2 capacity to uptake extracellular LDL. Indeed, BUO and OMN $(25.0 \mu \mathrm{g} / \mathrm{mL})$ improved the ability of HepG2 cells to absorb LDL-Dylight 550 up to $209 \pm 74 \%$ and $256 \pm 42 \%(p<0.01)$, respectively, whereas pravastatin $(1 \mu \mathrm{M})$ increased it by up to $215 \pm 30.8 \%(p<0.0001)$ (Figure 5).

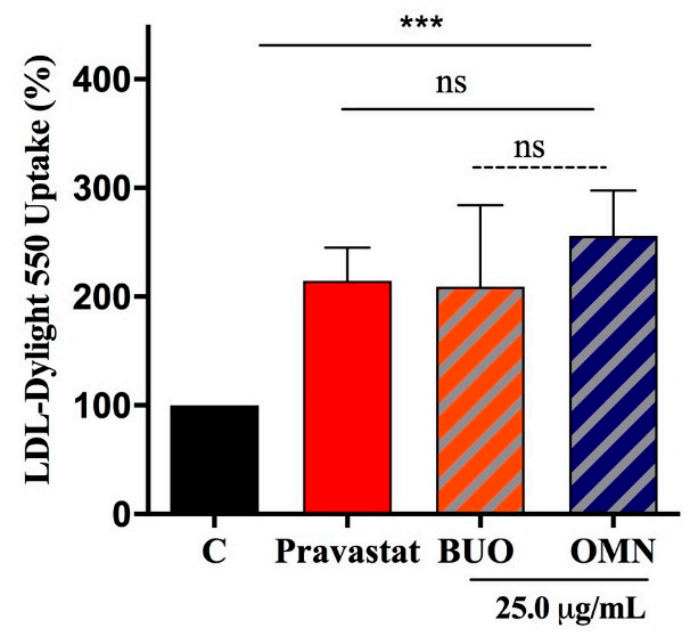

Figure 5. Fluorescent LDL-uptake assay after treatments of HepG2 with the phenol extracts. Both BUO and OMN increase the hepatic cell capability to uptake LDL from the extracellular environment. Experiments were carried out in parallel with pravastatin (Pravastat $1.0 \mu \mathrm{M}$ ) as positive control (B). Data represent the mean \pm s.d. of six independent experiments performed in triplicate. $\left.{ }^{* * *}\right) p<0.001$, ns: not significant. C. untreated HepG2 cells: pravastat: pravastatin.

\subsection{The Phenol Extracts Do Not Modulate the Mature PCSK9 Protein Level}

Both of the extracts were incapable of modulating the mature PCSK9 protein levels and they were also ineffective on the activation of HNF1- $\alpha$, the PCSK9 transcription factor (Figure 6A,B). On the contrary, pravastatin $(1 \mu \mathrm{M})$, upon the direct activation of HNF1- $\alpha$ up to $118 \pm 3.8 \%(p<0.05)$, significantly increased the PCSK9 protein level up to $125 \pm 3.4 \%(p<0.01)$ (Figure 6A,B). 
A
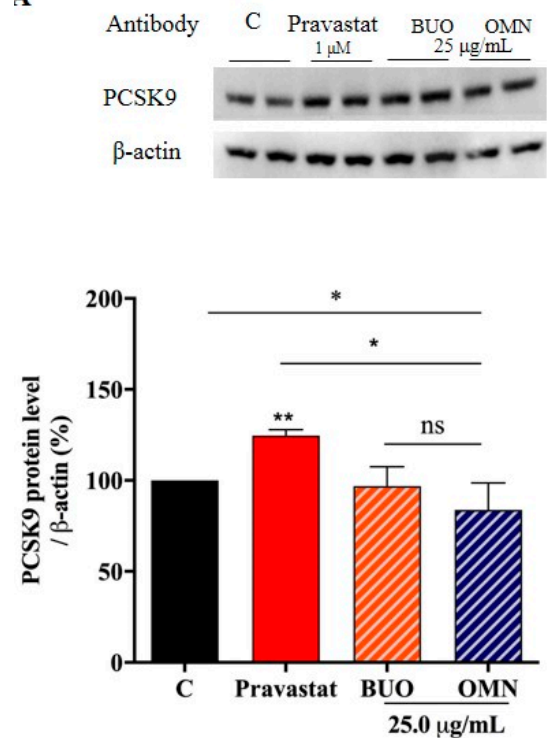

B
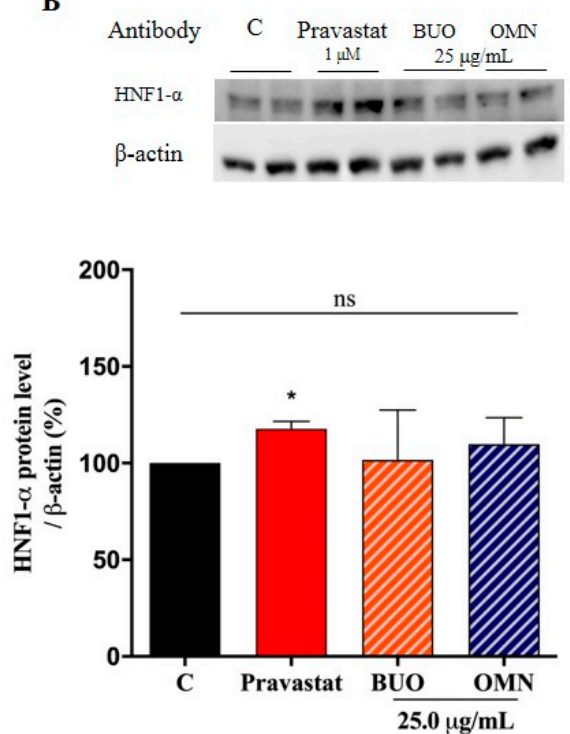

Figure 6. Effects of the phenol extracts on the PCSK9 pathway. BUO and OMN extracts did not affect the PCSK9 protein levels (A) and did not produce any effect on the modulation of HNF1- $\alpha(\mathbf{B})$. On the contrary, pravastatin (Pravastat 1. $\mu \mathrm{M}$ ) increases the PCSK9 protein level (A) due to the activation of the HNF1- $\alpha$ a, transcription factor $(\mathbf{B})$. Data represent the mean \pm s.d. of eight independent experiments performed in duplicate. $\left({ }^{*}\right) p<0.05,\left({ }^{*}\right) p<0.01$, ns: not significant. C. untreated HepG2 cells; Pravastat: pravastatin.

\section{Discussion}

EVOO is a rich source of phenols that are characterized by antioxidant activity, which counteracts the accumulation of free radicals and, therefore, has a positive effect on human health. Numerous studies have shown the beneficial EVOO effects in improving the lipid profile by increasing HDL-cholesterol and reducing LDL-cholesterol and triglyceride levels, reduces oxidative stress, and inhibits human lipoprotein oxidation, which makes LDL less atherogenic. In addition, the European Food Safety Authority (EFSA) approved the health claim for EVOO phenols, suggesting that their consumption protect LDL particles from oxidative damage.

Based on all these considerations, our study is focused on a deeper mechanistic investigation in order to find out how two EVOO extracts with different phenolic profiles may additionally modulate the cholesterol metabolism, trying to evaluate the role of the secoiridoid compounds, the main phenols in both extracts (Figure 7).

The results clearly indicate that BUO and OMN extracts are both able to inhibit the HMGCoAR enzyme in a statistically significant way and with a dose dependent manner (Figure 1A,B). These results are in line with a previous study showing that the hepatic HMGCoAR activity is significantly decreased in rats fed a standard diet containing 1\% of cholesterol (control diet) enriched with an EVOO phenolic extract versus control rats only fed the control diet [24].

These findings prompted us to investigate in a detailed way the cellular modulation of the LDLR pathway upon HMGCoAR inhibition. The cellular study was carried out on HepG2 cells, because the hepatocyte is the major cell that is involved in the clearance of plasma LDL cholesterol through the LDLR activity. Preliminary MTT experiments were performed in order to exclude any potential cytotoxic effect of both BUO and OMN samples, showing that both EVOO extracts are safe for HepG2 cells up to $100 \mu \mathrm{g} / \mathrm{mL}$. Based on these results, HepG2 cells were treated with $25.0 \mu \mathrm{g} / \mathrm{mL}$ of each phenol extract, i.e., at a concentration that was about 10-fold lower than the smallest cytotoxic dose and that is a compromise dose between the in vitro HMGCoAR activity assay results and the cytotoxic effects exerted by EVOO extracts on HepG2 cells, respectively. Thus, immunoblotting experiments were assessed in order to investigate the effects on the key targets that are involved in the LDLR pathway. 


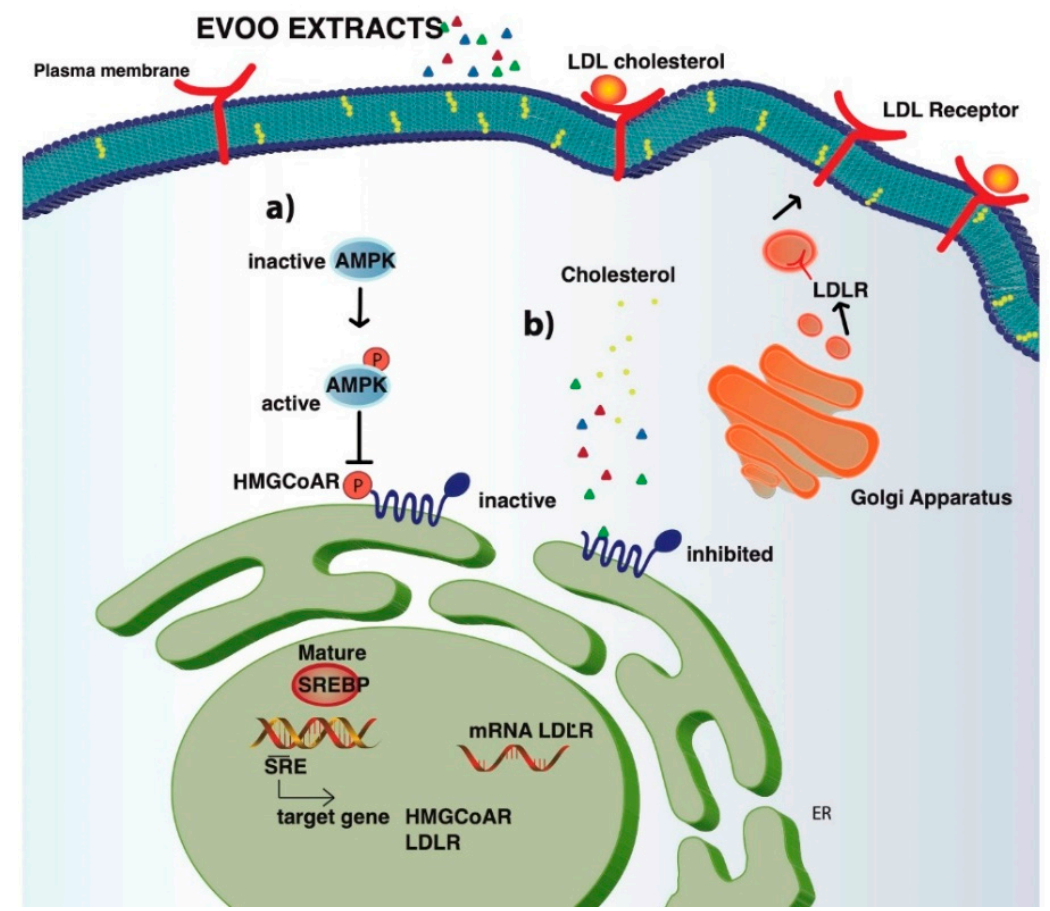

Figure 7. Potential EVOO extracts mechanism of action. Upon cell penetration, EVOO extracts (BUO and OMN) act as competitive inhibitor of HMGCoAR leading to a reduction of intracellular cholesterol synthesis. When intracellular cholesterol level decreases, the transcription factor SREBP2 is activated and LDLR and HMGCoAR genes are transcripted with subsequent increase of LDLR and HMGCoAR protein levels and localization of LDLR in plasma membrane (a). In parallel, evidences suggest that in a synergic way, BUO and OMN are able to reduce the cholesterol level production through AMPK-pathway. In particular, both EVOO extracts activate AMPK through an increase of phosphorylation at Thr172, which in turn leads to an inactivation of its target substrate HMGCoAR through phosphorylation at serine $872(\mathrm{~b})$. For this reason, the distinct modulation of the two pathways leads to an increase of the LDLR activity, which can bind and carry the extracellular LDL in HepG2 cells with final hypocholesterolaemic effects.

Similarly, to $1 \mu \mathrm{M}$ pravastatin (the positive control), by inhibiting the HMGCoAR activity, these extracts modulate the intracellular cholesterol pathway, leading to an increase of the LDLR and HMGCoAR protein levels through the modulation of SREBP-2 protein level (Figures 3 and 4). The regulation of HMGCoAR is achieved at several levels: transcription, translation, and post-translation (degradation and phosphorylation) levels, respectively [3]. The HMGCoAR transcription and translation increase when the concentrations of the products of the mevalonate pathway are low and decrease when the sterol concentrations are high [25]. The short-term regulation of HMGCoAR is obtained by inhibition through the phosphorylation of Ser872 by AMPK pathway activation. Indeed, the enzyme is physiologically present in the cell in an active non-phosphorylated form $(30 \%)$ and an inactive phosphorylated one (70\%). AMPK is a kinase that plays a key role in cellular energy homeostasis, largely to activate glucose and fatty acid uptake and oxidation when cellular energy is low. Statins are able to activate AMPK, with the consequence of a synergistic inhibition of HMGCoAR activity [26]. In this context, our results show that both phenol extracts induce AMPK activation through its phosphorylation at the Thr172 residue, which, in turn, determines an inhibition of HMGCoAR activity (Figure 4B,C). Indeed, this activation produced an increase of the HMGCoAR phosphorylation levels at Ser872 residue (AMPK phosphorylation site). Therefore, the ratio between pHMGCoAR/total HMGCoAR was calculated for both treated and untreated cells (Figure $4 \mathrm{D}$ ). When the ratio of treated cells is higher than the ratio of untreated ones, it means that the enzyme is inactivated [27]. Taking all these considerations into account, EVOO phenols extracts are 
able not only to act as competitive inhibitors of the HMGCoAR in vitro, as suggested by the results that were obtained using in vitro purified catalytic domain of the enzyme, but also to inhibit the cellular HMGCoAR activity by enhancing AMPK activation. As far as the phenolic profiles are concerned, both $\mathrm{BUO}$ and OMN extracts are derived from EVOOs that are rich in secoiridoid derivatives of oleuropein and ligstrosides, which may be suggested as being mainly responsible for the observed effects.

Contextually, the modulation of PCSK9 intracellular processing was also investigated. Both SREBP-2 and HNF1- $\alpha$ have been identified as transcriptional activators of PCSK9 gene expression, but only SREBP-2 is able to control the LDLR expression. It is important to observe that statins enhance the level of PCSK9, leading to an increase in the degradation of LDLR, which might be seen as a compensation mechanism that balances the increase of the production of the LDLR [28,29]. In agreement with literature, upon activation of $\mathrm{HNF} 1-\alpha$, pravastatin increases the mature PCSK9 protein levels (Figure 6). On the contrary, instead, neither BUO nor OMN extracts $(25.0 \mu \mathrm{g} / \mathrm{mL})$ were able to influence the HNF1- $\alpha$ protein level and, as a direct consequence, they did not modulate the mature PCSK9 protein levels (Figure 6). It is certainly a main outcome of our work to have demonstrated that EVOO phenols are able to ameliorate the cholesterol profile without presenting this relevant drawback of statins.

The ability of both phenol extracts to positively modulate the LDLR protein levels on the hepatocyte cellular surface was assessed using an ICW assay, i.e., a quantitative colorimetric cell-based assay that allows the detection of target proteins in fixed cultured cells directly in microplates, using a target-specific primary antibody and a horseradish peroxidase-conjugated secondary antibody [22]. In agreement with the ICW results, the treatment with both EVOO phenol extracts improves the ability of HepG2 cells to absorb fluorescent LDL from the extracellular environment, which suggests that this LDLR increase is linked to the functional ability to decrease the cholesterol level from the extracellular environment (Figure 5).

As indicated in the introduction, another objective of the work was to compare the bioactivities of the BUO and OMN extracts whose phenolic compositions are diverse (Tables 1 and 2). Apparently, the different phenol profile only marginally influences their behaviors, since they share the same mechanism of action at the molecular level.

In light of these results, it seems possible to affirm that all of the secoiridoid derivatives from the precursors oleuropein and ligstroside are suitable for inducing the same biological effects, which suggests a similar role for the hydroxytyrosol and tyrosol derivatives. This result is partially new considering that only recently it has been confirmed that tyrosol derivatives are more abundant than those of hydroxytyrosol in most EVOOs $[16,30]$, unlike what is indicated in previous literature.

\section{Conclusions}

This study contributes to clarify the mechanism behind the protective effects of EVOO phenols on the cardiovascular system, which suggests a role played not only by hydroxytyrosol, but also by the entire pool of secoiridoid molecules. Indeed, the molecular mechanisms of these EVOO phenol extracts may explain, at least in part, the hypocholesterolemic activity observed so far in many studies that were carried out on the EVOO.

Supplementary Materials: The following documents are available online at http://www.mdpi.com/2072-6643/12/ 6/1723/s1, Brief description of material and methods.

Author Contributions: Conceptualization, C.L.; Funding acquisition, M.L.C., F.C., A.A. and N.M.; Investigation, C.L., M.B. (Maria Bellumori), L.C., M.B. (Martina Bartolomei) and C.B.; Supervision, C.L. and N.M.; Writing-original draft, C.L., M.B. (Maria Bellumori) and L.C.; Writing-review \& editing, C.L., M.L.C., F.C., A.A. and N.M. All authors have read and agreed to the published version of the manuscript.

Funding: This research was funded by COMPETiTiVE-Claims of Olive oil to iMProvE the market ValuE project (AGER 2 Project) grant number 2016-0174.

Acknowledgments: We are indebted to Carlo Sirtori Foundation (Milan, Italy) for having provided part of equipment used in this experimentation 
Conflicts of Interest: The authors declare no conflicts of interest.

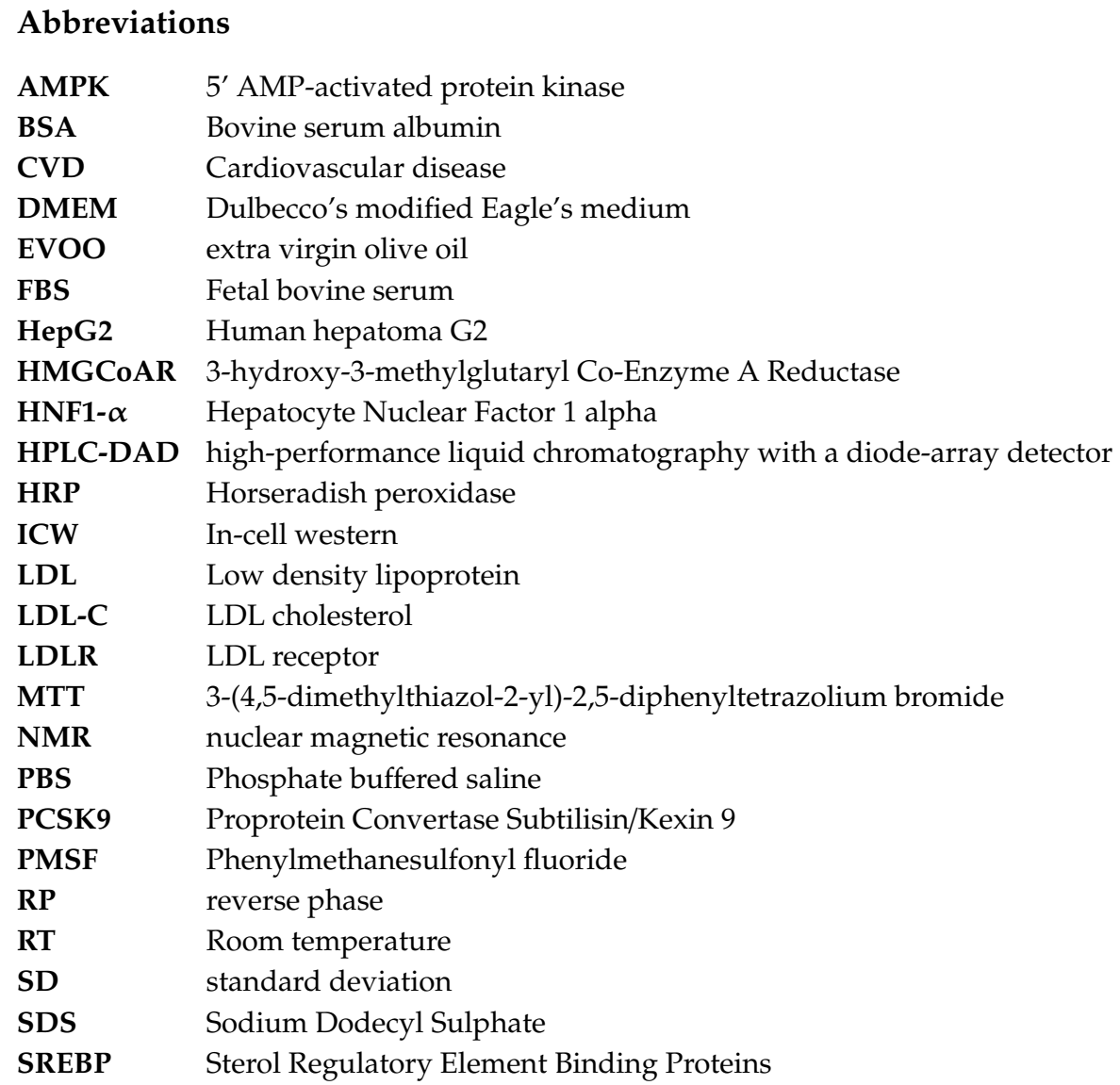

\section{References}

1. Brown, M.S.; Goldstein, J.L. Lipoprotein receptors in the liver. Control signals for plasma cholesterol traffic. J. Clin. Investig. 1983, 72, 743-747. [CrossRef] [PubMed]

2. Gu, H.M.; Zhang, D.W. Hypercholesterolemia, low density lipoprotein receptor and proprotein convertase subtilisin/kexin-type 9. J. Biomed. Res. 2015, 29, 356-361. [PubMed]

3. Goldstein, J.L.; Brown, M.S. Regulation of the mevalonate pathway. Nature 1990, 343, 425-430. [CrossRef]

4. Brown, M.S.; Goldstein, J.L. The SREBP pathway: Regulation of cholesterol metabolism by proteolysis of a membrane-bound transcription factor. Cell 1997, 89, 331-340. [CrossRef]

5. Horton, J.D.; Goldstein, J.L.; Brown, M.S. SREBPs: Activators of the complete program of cholesterol and fatty acid synthesis in the liver. J. Clin. Investig. 2002, 109, 1125-1131. [CrossRef]

6. Lagace, T.A.; Curtis, D.E.; Garuti, R.; McNutt, M.C.; Park, S.W.; Prather, H.B.; Horton, J.D. Secreted PCSK9 decreases the number of LDL receptors in hepatocytes and in livers of parabiotic mice. J. Clin. Investig. T 2006, 116, 2995-3005. [CrossRef]

7. Park, S.W.; Moon, Y.A.; Horton, J.D. Post-transcriptional regulation of low density lipoprotein receptor protein by proprotein convertase subtilisin/kexin type 9a in mouse liver. J. Biol. Chem. 2004, 279, 50630-50638. [CrossRef]

8. Seidah, N.G.; Prat, A. The biology and therapeutic targeting of the proprotein convertases. Nat. Rev. Drug Discov. 2012, 11, 367-383. [CrossRef]

9. Chaudhary, R.; Garg, J.; Shah, N.; Sumner, A. PCSK9 inhibitors: A new era of lipid lowering therapy. World J. Cardiol. 2017, 9, 76-91. [CrossRef] [PubMed]

10. Perona, J.S.; Cañizares, J.; Montero, E.; Sánchez-Domínguez, J.M.; Catalá, A.; Ruiz-Gutiérrez, V. Virgin olive oil reduces blood pressure in hypertensive elderly subjects. Clin. Nutr. 2004, 23, 1113-1121. [CrossRef] [PubMed] 
11. Covas, M.I.; Nyyssönen, K.; Poulsen, H.E.; Kaikkonen, J.; Zunft, H.J.F.; Kiesewetter, H.; Nascetti, S. The effect of polyphenols in olive oil on heart disease risk factors: A randomized trial. Ann. Intern. Med. 2006, 145, 333-341. [CrossRef]

12. Carluccio, M.A.; Massaro, M.; Scoditti, E.; De Caterina, R. Vasculoprotective potential of olive oil components. Mol. Nutr. Food Res. 2007, 51, 1225-1234. [CrossRef] [PubMed]

13. Berrougui, H.; Cloutier, M.; Isabelle, M.; Khalil, A. Phenolic-extract from argan oil (Argania spinosa L.) inhibits human low-density lipoprotein (LDL) oxidation and enhances cholesterol efflux from human THP-1 macrophages. Atherosclerosis 2006, 184, 389-396. [CrossRef] [PubMed]

14. Marcelino, G.; Hiane, P.A.; Freitas, K.D.C.; Santana, L.F.; Pott, A.; Donadon, J.R.; \& Guimarães, R.D.C.A. Effects of Olive Oil and Its Minor Components on Cardiovascular Diseases, Inflammation, and Gut Microbiota. Nutrients 2019, 11, 1826. [CrossRef]

15. EU Regulation 1989. Off. J. Eur. Com. 2003, L295, 57-77. Available online: https://eur-lex.europa.eu/legalcontent/EN/TXT/?uri=celex:32003R1989 (accessed on 1 April 2019).

16. Bellumori, M.; Cecchi, L.; Innocenti, M.; Clodoveo, M.L.; Corbo, F.; Mulinacci, N. The EFSA Health Claim on Olive Oil Polyphenols: Acid Hydrolysis Validation and Total Hydroxytyrosol and Tyrosol Determination in Italian Virgin Olive Oils. Molecules 2019, 24, 2179. [CrossRef]

17. IOC/T.20/Doc No. 29, Official Method of Analysis. Determination of Biophenols in Olive oil by HPLC. International Olive Council, Madrid. 2009. Available online: http://www.internationaloliveoil.org/estaticos/ view/224-testing-methods (accessed on 1 April 2019).

18. Bellumori, M.; Cecchi, L.; Romani, A.; Mulinacci, N.; Innocenti, M. Recovery and stability over time of phenolic fractions by an industrial filtration system of olive mill wastewaters: A three-year study. J. Sci. Food Agric. 2018, 98, 2761-2769. [CrossRef]

19. Karkoula, E.; Skantzari, A.; Melliou, E.; Magiatis, P. Direct measurement of oleocanthal and oleacein levels in olive oil by quantitative (1)H NMR. Establishment of a new index for the characterization of extra virgin olive oils. J. Agric. Food Chem. 2012, 60, 11696-11703. [CrossRef]

20. Zanoni, C.; Aiello, G.; Arnoldi, A.; Lammi, C. Investigations on the hypocholesterolaemic activity of LILPKHSDAD and LTFPGSAED, two peptides from lupin beta-conglutin: Focus on LDLR and PCSK9 pathways. J. Funct. Foods 2017, 32, 1-8. [CrossRef]

21. Lammi, C.; Zanoni, C.; Calabresi, L.; Arnoldi, A. Lupin protein exerts cholesterol-lowering effects targeting PCSK9: From clinical evidences to elucidation of the in vitro molecular mechanism using HepG2 cells. J. Funct. Foods 2016, 23, 230-240. [CrossRef]

22. Lammi, C.; Zanoni, C.; Arnoldi, A. A simple and high-throughput in-cell Western assay using HepG2 cell line for investigating the potential hypocholesterolemic effects of food components and nutraceutics. Food Chem. 2015, 169, 59-64. [CrossRef] [PubMed]

23. Zanoni, C.; Aiello, G.; Arnoldi, A.; Lammi, C. Hempseed peptides exert hypocholesterolemic effects with a statin-like mechanism. J. Agric. Food Chem. 2017, 65, 8829-8838. [CrossRef] [PubMed]

24. Benkhalti, F.; Prost, J.; Paz, E.; Perez-Jimenez, F.; El Boustani, E. Effects of feeding virgin olive oil or their polyphenols on lipid of rat liver. Nutr. Res. 2002, 22, 1067-1075. [CrossRef]

25. Istvan, E.S.; Palnitkar, M.; Buchanan, S.K.; Deisenhofer, J. Crystal structure of the catalytic portion of human HMG-CoA reductase: Insights into regulation of activity and catalysis. EMBO J. 2000, 19, 819-830. [CrossRef]

26. Sun, W.; Lee, T.S.; Zhu, M.; Gu, C.; Wang, Y.; Zhu, Y.; Shyy, J.Y. Statins activate AMP-activated protein kinase in vitro and in vivo. Circulation 2006, 114, 2655-2662. [CrossRef]

27. Levy, E.; Ouadda, A.B.D.; Spahis, S.; Sane, A.T.; Garofalo, C.; Grenier, É.; Ménard, D. PCSK9 plays a significant role in cholesterol homeostasis and lipid transport in intestinal epithelial cells. Atherosclerosis 2013, 227, 297-306. [CrossRef]

28. Careskey, H.E.; Davis, R.A.; Alborn, W.E.; Troutt, J.S.; Cao, G.; Konrad, R.J. Atorvastatin increases human serum levels of proprotein convertase subtilisin/kexin type 9. J. Lipid Res. 2008, 49, 394-398. [CrossRef] 
29. Welder, G.; Zineh, I.; Pacanowski, M.A.; Troutt, J.S.; Cao, G.; Konrad, R.J. High-dose atorvastatin causes a rapid sustained increase in human serum PCSK9 and disrupts its correlation with LDL cholesterol. J. Lipid Res. 2010, 51, 2714-2721. [CrossRef]

30. Tsimidou, M.Z.; Sotiroglou, M.; Mastralexi, A.; Nenadis, N.; García-González, D.L.; Gallina Toschi, T. In House Validated UHPLC Protocol for the Determination of the Total Hydroxytyrosol and Tyrosol Content in Virgin Olive Oil Fit for the Purpose of the Health Claim Introduced by the EC Regulation 432/2012 for “Olive Oil Polyphenols”. Molecules 2019, 24, 1044. [CrossRef]

(C) 2020 by the authors. Licensee MDPI, Basel, Switzerland. This article is an open access article distributed under the terms and conditions of the Creative Commons Attribution (CC BY) license (http://creativecommons.org/licenses/by/4.0/). 